\title{
A New Formula for Conjugate Gradient in Unconstrained Optimization
}

\author{
Hussein A. Wali \\ ha678413@gmail.com
}

\author{
Khalil K. Abbo \\ kh_196538@yahoo.com
}

Department of Mathematics

College of Computers Sciences and Mathematics

University of Mosul, Mosul, Iraq

Received on: 25/08/2019

Accepted on: 23/09/2019

\begin{abstract}
The conjugate gradient method is an important part of the methods of optimization that are not constrained by local convergence characteristics. In this research, a new formula for the conjugated coefficient is derived depending on the linear structure. The new method fulfills the regression requirement. In addition, using the Wolff search line terms, the overall convergence of the new method has been demonstrated. At the end of the research were presented numerical results that show the effectiveness of the proposed method.
\end{abstract}

Keywords: unconstrained optimization, conjugate gradient method, sufficient descent property.

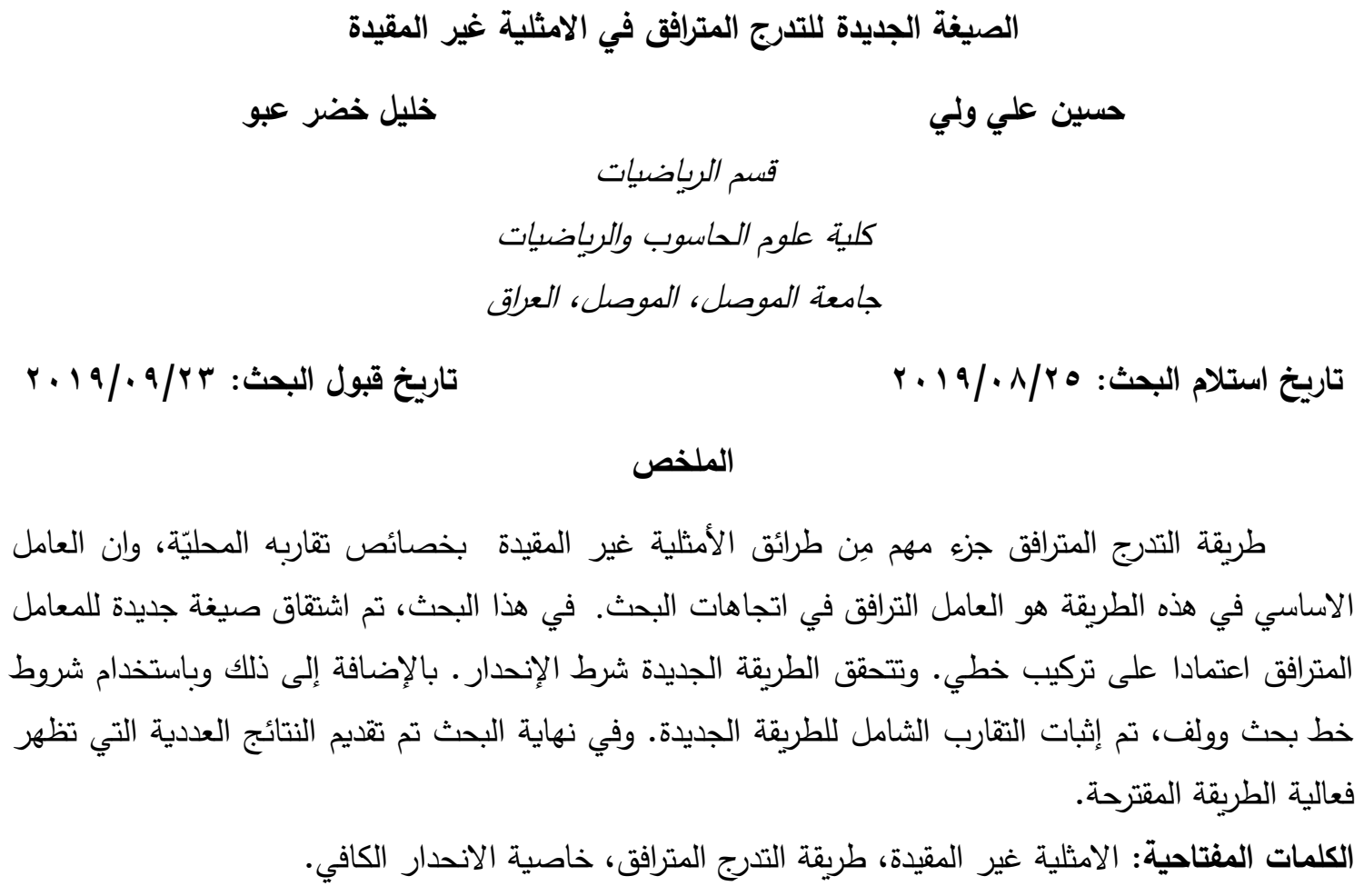


من الصفات المهمة أن طريقة التدرج المترافق لها القابلية على توليد مجموعة متجهات لها خاصية الترافق

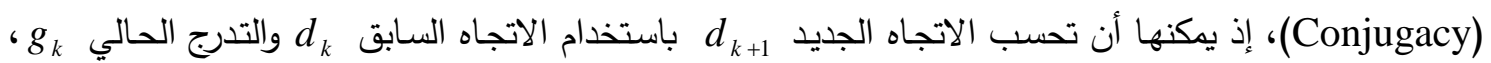

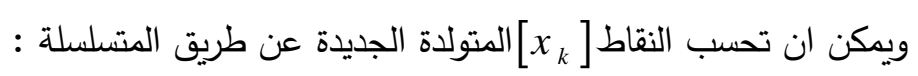

$$
x_{k+1}=x_{k}+\alpha_{k} d_{k}, k=1,2, \ldots
$$

ويختار بحيث يكون تركيباً خطياً من( جميع الاتجاهات السابقة

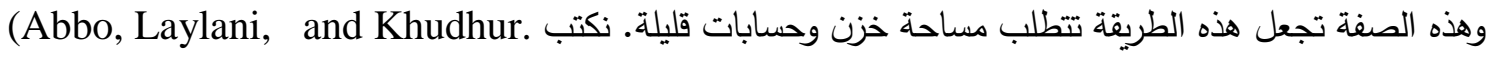

$$
d_{k+1}=-g_{k+1}+\beta_{k+1} d_{k}
$$

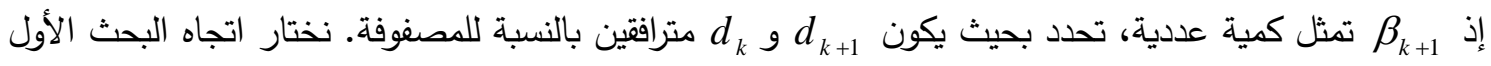
عند النقطة الابتدائية التقارب لطرائق التدرج المترافق يكون خطياً ما لم يسترجع التكرار من أكثر الصيخ شهرة. الخيارات المختلفة للمعلمة ${ }^{\prime} \beta_{k+1}$

$$
\begin{gathered}
\beta_{k+1}^{H S}=\frac{g_{k+1}^{T} y_{k}}{d_{k}^{T} y_{k}} \\
\beta_{k+1}^{F R}=\frac{g_{k+1}^{T} g_{k}}{g_{k}^{T} g_{k}} \\
\beta_{k+1}^{P R}=\frac{g_{k+1}^{T} y_{k}}{g_{k}^{T} g_{k}} \\
\beta_{k+1}^{D Y}=\frac{g_{k+1}^{T} g_{k+1}}{d_{k}^{T} y_{k}} \\
\beta_{k}^{C C}=\left(1-\theta_{k}\right) \frac{g_{k+1}^{T} y_{k}}{g_{k}^{T} g_{k}}+\theta_{k} \frac{g_{k+1}^{T} g_{k+1}}{s_{k}^{T} y_{k}} \\
\frac{\left(y_{k+1}^{T} g_{k+1}\right)\left(s_{k}^{T} y_{k}\right)-\left(y_{k+1}^{T} g_{k+1}\right)\left(g_{k}^{T} g_{k}\right)}{\left(y_{k+1}^{T} g_{k+1}\right)\left(s_{k}^{T} g_{k}\right)-\left(g_{k+1}^{T} g_{k+1}\right)\left(g_{k}^{T} g_{k}\right)} \\
\beta_{k}^{N D}=\left(1-\theta_{k}\right) \frac{g_{k+1}^{T} y_{k}}{g_{k}^{T} g_{k}}+\theta_{k} \frac{g_{k+1}^{T} g_{k+1}}{s_{k}^{T} y_{k}} \\
\theta_{k}=\frac{g_{k+1}^{T} s_{k}}{g_{k}^{T} g_{k+1}} \\
\beta_{k+1}^{K H 2}=\frac{\left(g_{k+1}^{T} y_{k}\right)^{2}}{g_{k}^{T} g_{k}\left(2 g_{k+1}^{T} y_{k}-\left\|g_{k+1}\right\|^{2}\right)} \\
\left(g_{k+1}^{T} y_{k}\right)^{2} \\
\beta_{k}^{T} y_{k}\left(2 g_{k+1}^{T} y_{k}-\left\|g_{k+1}\right\|^{2}\right)
\end{gathered}
$$

(Hestenes-Stiefel(HS)1952)

(Fletcher-Reeves (FR) 1964)

(Polak-Ribière (PR) 1969)

(Dai-Yuan (DY) 1999)

(Andrei, 2007a )

( Andrei, 2007d)

(Abbo and Khudhur, 2015a)

(Abbo and Khudhur, 2015b)

الطرائق الهجينة هي الربط بين طريقتين إحداها تمتلك خواص حسابية جيدة Good Computational) فالخاخى تمتلك خواص تقارب شاملة قوية(Strong Convergence Properties ). وعليه فالخوارزمية الهجينة لها حافز قوي للاستفادة من السمات البارزة لخوارزمية CG الكلاسيكية (التقليدية). 
(Andrei, 2011). هناك العديد من الاقتراحات من الطرائق الهجينة من اجل الوصول الى افضل طرائق التدرج

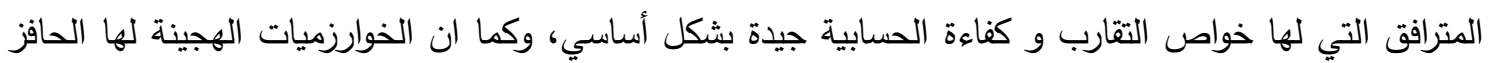

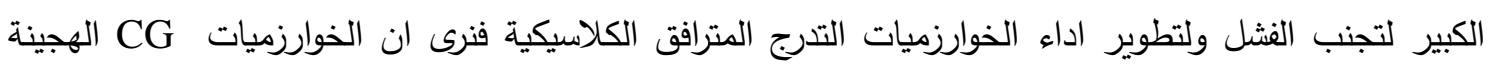
(Andrei, 2009a). احسن من الخوارزميات التدرج المترافق الكلاسيكية ولطوير اختلاف معامل الترافق طرائق التدرج المترافق المختلفة لحل مسائل الامثلية اللاخطية وغير المقيدة.

2 اشتقاق الصيغة الجديدة للتدرج المترافق The Derivation of the New CG Formals) الفكرة من هذا الاشتقاق هو تطوير طريقة Dai and Lia اعتماداً على تركيب خطي. ان العامل الاساسي في طرائق التدرج المترافق هو العامل الترافق الاتجاهات البحث $d_{k}^{T} H_{j} d_{k}=0, \forall k \neq j$

نلاحظ ان المعادلة اعلاه تكون صحيحة في حالة دالة الهدف دالة تربيعية محدبة وبحث خطي تام , يمكن اعادة $d_{k+1}^{T} y_{k}=0$

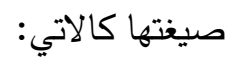
وفي العام 1978 قد تم تعميم الشرط الترافق (6erry Pe بالصيغةالاتية: $d_{k+1}^{T} y_{k}=-g_{k+1}^{T} s_{k}$

قام العالمان Dai And Laio في السنة 2001 بالتعميم الثرط الترافق المقترح من قبل Perry الى الصيغة

$$
d_{k+1}^{T} y_{k}=-t g_{k+1}^{T} s_{k}, t>0
$$$$
\beta_{k}=\frac{\mathrm{g}_{k+1}^{T}\left(y_{k}-t_{k} s_{k}\right)}{d_{k}^{T} y_{k}}
$$

وعلى اساسها اقترح معامل الترافق التالية:

$$
\beta_{k}=\frac{\mathrm{g}_{k+1}^{T}\left((1-\mathrm{r}) y_{k}-r s_{k}\right)}{d_{k}^{T} y_{k}}
$$

الان, اعتماداً على تركيب محدب نقترح الصيغة التالية:

$$
d_{k+1}=-g_{k+1}+\frac{g_{k+1}^{T}\left((1-r) y_{k}-r s_{k}\right)}{s_{k} y_{k}} s_{k}
$$

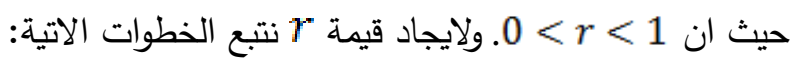

$$
\begin{aligned}
& d_{k+1}^{T} y_{k}=-g_{k+1}^{T} y_{k}+\left(\frac{\left((1-r) y_{k}^{T} g_{k+1}-r s_{k}^{T} g_{k+1}\right)}{s_{k}^{T} y_{k}}\right) s_{k}^{T} y_{k} \\
& \text { مع بعض العمليات الجبرية وتعويض عن } \\
& -g_{k+1}^{T} y_{k}+(1-r) y_{k}^{T} g_{k+1}-r s_{k}^{T} g_{k+1}=-s_{k}^{T} g_{k+1} \\
& -y_{k}^{T} g_{k+1}+y_{k}^{T} g_{k+1}-r y_{k}^{T} g_{k+1}-r s_{k}^{T} g_{k+1}=-s_{k}^{T} g_{k+1} \\
& \text { من المعادلة اعلاه نحصل على الاتي: } \\
& \text { نضرب المعادلة اعلاة في (y) }
\end{aligned}
$$




$$
\mathrm{r}=\frac{s_{k}^{T} \mathrm{~g}_{\mathrm{k}+1}}{\left(y_{k}^{T} \mathrm{~g}_{\mathrm{k}+1}+s_{k}^{T} \mathrm{~g}_{\mathrm{k}+1}\right)}
$$

خوارزمية (2.1) (KHC Algorithm )

الخطوة 1 : اختر قيمة ابتدائية

الخطوة 2 : إذا كان 8 : الخطوة 3: احسب طول الخطوة 0 ا مolfe يحقق شرطي

$$
\begin{gathered}
f\left(x_{k}+\alpha_{k} d_{k}\right) \leq f\left(x_{k}\right)+c_{1} \alpha_{k} \nabla f\left(x_{k}\right)^{T} d_{k} \\
\left|\nabla f\left(x_{k}+\alpha_{k} d_{k}\right)^{T} d_{k}\right| \leq c_{2}\left|\nabla f\left(x_{k}\right)^{T} d_{k}\right|,
\end{gathered}
$$

الخطوة 4: احسب

الخطوة 5: احسب اتجاه البحث الخطوة 6: إذا كان معدل التقارب $\alpha_{k+1}=\alpha_{k}\left(\frac{\left\|d_{k}\right\|}{\left\|d_{k+1}\right\|}\right)$ الخطوة 7 : احسب قيمة ابتدائية

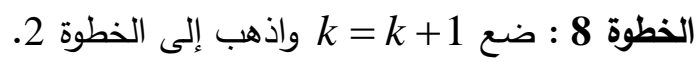
3 بعض الخواص النظرية للخوارزمية الجديدة

نبرهنها مثل خاصية الأنحدار من هذهِ الخوارزمية وخاصية الترافق.

(3.1) (النظرية (برهن

سوف نفرض الفرضيات التالية على دالة الهدف

(i) مستوى المجموعة (Level Set) هناك ثابت موجب

(ii)

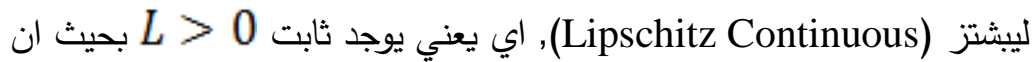
$\|g(x)-g(y)\| \leq L\|x-y\|, \forall x, y \in N$

$$
\begin{aligned}
& \text { باستخدام الفرضية , نحصل على الاتي: } \\
& \text { يوجد ثابت > > ب بحيث ان }
\end{aligned}
$$

$\|\nabla f(x)\| \leq \Gamma, \forall x \in S$

.(Laylani, , Abbo, and Khudhur. 2018), (Jabbar, Abbo and Khudhur. 2018)

4 خاصية الأنحار للصيغة الجديدة (The Descent Property of the new formula) سوف نبرهن خاصية الانحدار الكافي (Sufficient Descent Property) للصيغة الجديدة المقترحة لخوارزمية التدرج المترافق ويعبرعن خاصية الانحدار الكافي لخوارزمية التدرج المترافق بالصيغة التالية : $g_{k+1}^{T} d_{k+1} \leq-c\left\|g_{k+1}\right\|^{2}$ for $k \geq 0$ and $c>0$

ومن جهة اخرى, يحقق لنا شرط (Lipschize) 
$s_{k}^{T} y_{k} \leq L\left\|s_{k}\right\|^{2}$

(Laylani, , Abbo, and Khudhur. 2018)

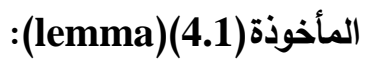

نفرض ان الفرضيتين (ii),(ii) في النظرية (3.1) قد تحقت. لذا

$g_{k}^{T} g_{k-1} \geq 0$

البرهان:

$g_{k}=\left(g_{i}\left(x_{k}\right)\right), i=1,2, \ldots, n$

لتكن g

اذا فرضنا ان لدينا حالتين كالاتي:

اولا: اذا كانت اما ثانيا: اذا كانت اما

اذا كانت : $\left|g_{i}\left(x_{k}\right)-g_{i}\left(x_{k-1}\right)\right|<\varepsilon$ $-\varepsilon+g_{i}\left(x_{k-1}\right)<g_{i}\left(x_{k}\right)$

ويؤدي الى ان

․ لدينا تتاقض ان

اذا كانت $\quad \forall \varepsilon>0$.(Liptchiz) مستمرة $\quad g_{k} g \quad g_{i}\left(x_{k}\right)>0, g_{i}\left(x_{k-1}\right)<0 \quad$ بحيث $\left|g_{i}\left(x_{k}\right)-g_{i}\left(x_{k-1}\right)\right|<\varepsilon$ اذن ع

النظربة (4.2):

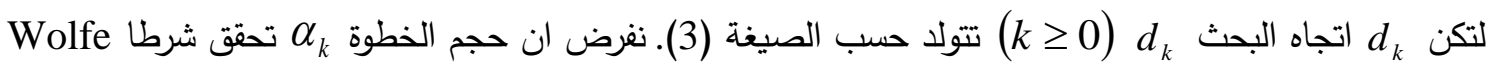
القياسي عندئذٍ $d_{k}$ تحقق خاصية الأنحدار الكافي (5). البرهان:

برهان بطريقة الاستقراء الرياضي:

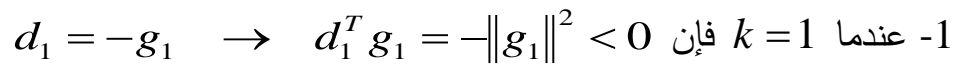

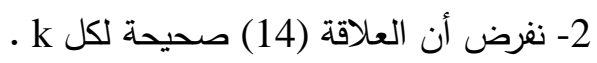

3- نبرهن صحة العلاقة (5) عندما $d_{k+1}^{T} g_{k+1}=-g_{k+1}^{T} g_{k+1}+\left(\frac{(1-r) y_{k}^{T} g_{k+1}-r s_{k}^{T} g_{k+1}}{s_{k}^{T} y_{k}}\right) s_{k}^{T} g_{k+1}$

وتبسيط المعادلة اعلاه نحصل على

$d_{k+1}^{T} g_{k+1}=-\left\|g_{k+1}\right\|^{2}+\frac{(1-r) y_{k}^{T} g_{k+1} s_{k}^{T} g_{k+1}}{s_{k}^{T} y_{k}}-\frac{r\left(s_{k}^{T} g_{k+1}\right)^{2}}{s_{k}^{T} y_{k}}$

نهمل الجزء السالب من المعادلة اعلاه ونغرض ان (r $c=$ 


$$
\begin{aligned}
& d_{k+1}^{T} g_{k+1}=-\left\|g_{k+1}\right\|^{2}+c \frac{y_{k}^{T} g_{k+1} s_{k}^{T} g_{k+1}}{s_{k}^{T} y_{k}} \\
& \text { نستخدام الصيغة الاتية } \\
& d_{k+1}^{T} g_{k+1} \leq-\left\|g_{k+1}\right\|^{2}+c \frac{y_{k}^{T} g_{k+1} s_{k}^{T} y_{k}}{s_{k}^{T} y_{k}} \\
& d_{k+1}^{T} g_{k+1} \leq-\left\|g_{k+1}\right\|^{2}+c y_{k}^{T} g_{k+1} \\
& d_{k+1}^{T} g_{k+1} \leq-\left\|g_{k+1}\right\|^{2}+c\left(\mathrm{~g}_{k+1}-\mathrm{g}_{k}\right)^{T} g_{k+1} \\
& d_{k+1}^{T} g_{k+1} \leq-\left\|g_{k+1}\right\|^{2}+c\left\|g_{k+1}\right\|^{2}-g_{k}^{T} g_{k+1}
\end{aligned}
$$

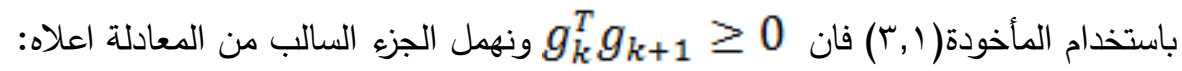

$$
\begin{aligned}
& d_{k+1}^{T} g_{k+1} \leq-\left\|g_{k+1}\right\|^{2}(1-c) \leq 0 \\
& \therefore d_{k+1}^{T} g_{k+1}<0
\end{aligned}
$$

5 تحليل التقارب (Convergence Analysis)

نبرهن في هذه الفقرة على أن طريقة KHC مع اتجاه البحث d d يتقارب تقارباً مطلقاً و لنبرهن ذلك نحتاج

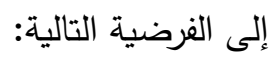

\section{(المأخوذة (5.1) ) (Lemma)}

سوف نفرض إن الفرضية (3.1) تتحقق، والمتتابعة $\} x_{k}$ نتولد من (2), علية إن d d الجاه بحث منحدر وطول الخطوة تحقق شرطا (Wolfe) الاعتياديين. إذ إن إن

$\sum_{k=0}^{\infty} \frac{\left(g_{k}^{T} d_{k}\right)^{2}}{\left\|d_{k}\right\|^{2}}<+\infty$

إذ إن المعادلة (15) تسمى شرط (Zoutendijk Condition) التي تعد على قدر من الأهمية لبرهان التقارب

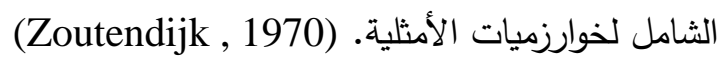

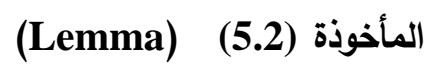

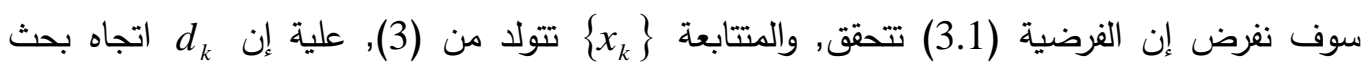
منحدر و $\alpha_{k}$ طول الخطوة تحقق شرطا (Wolfe) القويين

$f\left(x_{k}+\alpha_{k} d_{k}\right) \leq f\left(x_{k}\right)+c_{1} \alpha_{k} \nabla f\left(x_{k}\right)^{T} d_{k}$

$\left|\nabla f\left(x_{k}+\alpha_{k} d_{k}\right)^{T} d_{k}\right| \leq c_{2}\left|\nabla f\left(x_{k}\right)^{T} d_{k}\right|$,

$\sum_{k>1} \frac{1}{\left\|d_{k+1}\right\|^{2}}=\infty$ 


$$
\lim _{k \rightarrow \infty}\left(\inf \left\|g_{k}\right\|\right)=0
$$

الدالة الدحدبة بانتظام, لذلك من خلال المأخوذة (5.2) نستطيع ان نثبت النظريات الاتية. (Dai and Liao, 2001), (Khudhur. 2015)

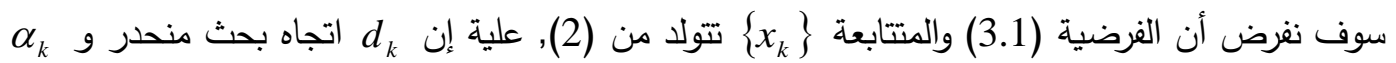

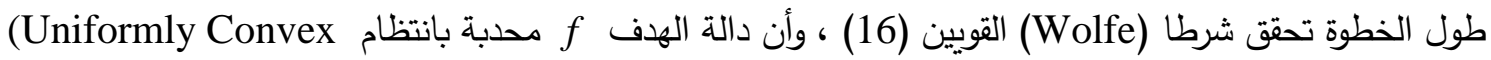

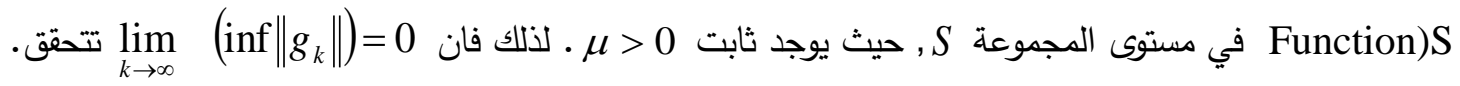
$\left\|d_{k+1}\right\|=\left\|-g_{k+1}+\left(\frac{(1-r) y_{k}^{T} g_{k+1}-r s_{k}^{T} g_{k+1}}{s_{k}^{T} y_{k}}\right) s_{k}\right\|$ $\left\|d_{k+1}\right\|=\left\|-g_{k+1}+\left(\frac{y_{k}^{T} g_{k+1}-r y_{k}^{T} g_{k+1}-r s_{k}^{T} g_{k+1}}{s_{k}^{T} y_{k}}\right) s_{k}\right\|$ $\left\|d_{k+1}\right\| \leq\left\|g_{k+1}\right\|+\frac{\left\|s_{k}\right\|^{2}\left\|g_{k+1}\right\|+(L-L r-r)}{\frac{1}{L\left\|s_{k}\right\|}}$

$\left\|d_{k+1}\right\| \leq\left\|g_{k+1}\right\|\left(1+1-r-\frac{r}{L}\right)$ $\left\|d_{k+1}\right\| \leq\left\|g_{k+1}\right\|\left(\frac{2 L-r L-r}{L}\right) \bar{y}_{k}$ $\sum_{k \geq 1} \frac{1}{\left\|d_{k}\right\|^{2}} \geq\left(\frac{1}{2 L-r L-r}\right)^{2} \frac{1}{\bar{y}} \sum_{k \geq 1} 1=\infty$

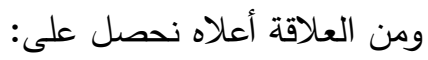
وباستخدام المأخوذة (5.2) فان (Numerical Results) النتائج العدادية 6

سنناقش في هذه الفقرة النتائج العددية للخوارزمية المقترحه الجديدة KHC التي حصلت عليها من استخدام

الصيغة الجديدة للمعامل الترافق لمجموعة من دوال الاختبار في الأمثلية غير المقيدة مأخوذة. (Andrei,2008)

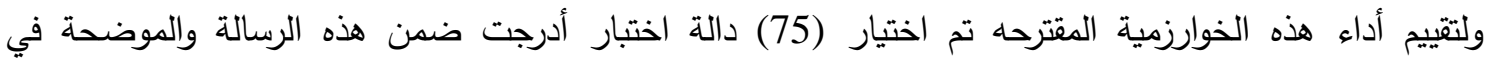
الملحق. تم اختيار الدوال للأبعاد 1000, ,.., , خوارزميات ND, CC الدقياس المستخدم لإيقاف تكرارات الخوارزميات هو بلغة فورتران (بالأعتماد على برنامج (Andrei) (version 6.6.0) (FORTRAN 77) ، إن دوال الاختبار تبدأ

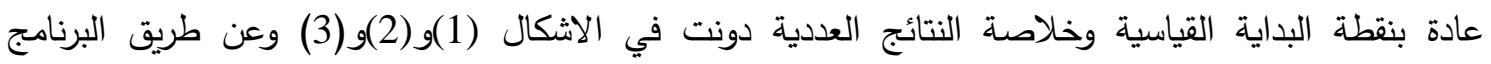
(Dolan and (Matlab R2009b) 


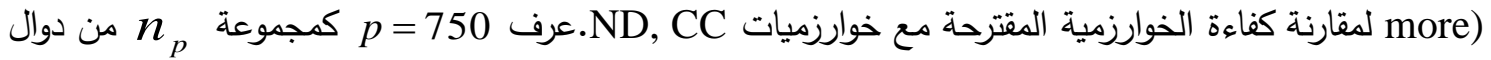
الاختبار و $4=4$ عدد الخوارزميات المستخدمة. لتكن $l_{p, s}$ يمثل عدد مرات إيجاد قيمة دالة الهدف من قبل الخوارزمية S د لحل مسألة

$$
r_{p, s}=\frac{l_{p, s}}{l_{p}^{*}}
$$

حيث ان

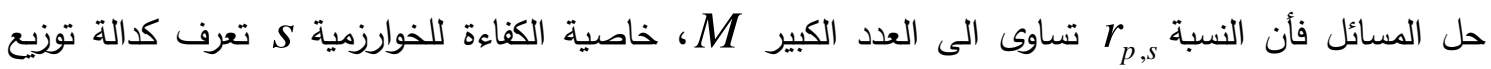
r المتراكم على نسبة الكفاءة

$$
\rho_{s}(\tau)=\frac{\operatorname{size}\left\{p \in P: r_{p, s} \leq \tau\right\}}{n_{p}}
$$

من الواضح (1) p pمثل النسبة المئوية لنجاح افضلية الخوارزمية S. خاصية الكفاءة يمكن أن تستخدم

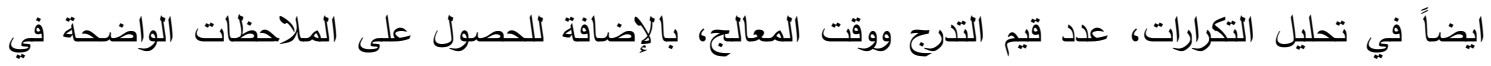
المخطط الاتي الاحداثيات الافقية ومقياس الاسسي، (E. D. Dolan and J. J. Mor'e.2001)

\begin{tabular}{|c|c|c|c|c|c|}
\hline No. & Function & Dim & Algor & iter & $\mathbf{F g}$ \\
\hline \multirow{6}{*}{1} & \multirow{3}{*}{$\begin{array}{c}\text { Extended } \\
\text { Trigonometric }\end{array}$} & \multirow{3}{*}{100} & $\mathrm{Cc}$ & 19 & 35 \\
\hline & & & $\mathrm{Nd}$ & 22 & 38 \\
\hline & & & $\mathrm{Kh}$ & 19 & 35 \\
\hline & \multirow{3}{*}{$\begin{array}{c}\text { Extended } \\
\text { Trigonometric }\end{array}$} & \multirow{3}{*}{1000} & $\mathrm{Cc}$ & 40 & 67 \\
\hline & & & $\mathrm{Nd}$ & 2002 & 2017 \\
\hline & & & $\mathrm{Kh}$ & 36 & 64 \\
\hline \multirow{6}{*}{2} & \multirow{3}{*}{$\begin{array}{l}\text { Extended } \\
\text { Penalty }\end{array}$} & \multirow{3}{*}{100} & $\mathrm{Cc}$ & 9 & 16 \\
\hline & & & $\mathrm{Nd}$ & 25 & 49 \\
\hline & & & $\mathrm{Kh}$ & 9 & 16 \\
\hline & \multirow{3}{*}{$\begin{array}{l}\text { Extended } \\
\text { Penalty }\end{array}$} & \multirow{3}{*}{1000} & $\mathrm{Cc}$ & 12 & 23 \\
\hline & & & $\mathrm{Nd}$ & 82 & 1870 \\
\hline & & & $\mathrm{Kh}$ & 12 & 23 \\
\hline \multirow{6}{*}{3} & \multirow{3}{*}{ Raydan 2} & \multirow{3}{*}{100} & $\mathrm{Cc}$ & 5 & 10 \\
\hline & & & $\mathrm{Nd}$ & 5 & 10 \\
\hline & & & $\mathrm{Kh}$ & 5 & 10 \\
\hline & \multirow{3}{*}{ Raydan 2} & \multirow{3}{*}{1000} & $\mathrm{Cc}$ & 5 & 10 \\
\hline & & & $\mathrm{Nd}$ & 5 & 10 \\
\hline & & & $\mathrm{Kh}$ & 5 & 10 \\
\hline \multirow{6}{*}{4} & & \multirow{3}{*}{100} & $\mathrm{Cc}$ & 11 & 20 \\
\hline & Extended Himmelblau & & $\mathrm{Nd}$ & 29 & 58 \\
\hline & & & $\mathrm{Kh}$ & 11 & 20 \\
\hline & \multirow{3}{*}{$\begin{array}{l}\text { Extended Himmelblau } \\
\text { HIMMELBC (CUTE) }\end{array}$} & \multirow{3}{*}{1000} & $\mathrm{Cc}$ & 23 & 36 \\
\hline & & & $\mathrm{Nd}$ & 31 & 62 \\
\hline & & & $\mathrm{Kh}$ & 12 & 22 \\
\hline \multirow{4}{*}{5} & \multirow{3}{*}{ Extended PSC1 } & \multirow{3}{*}{100} & $\mathrm{Cc}$ & 9 & 18 \\
\hline & & & $\mathrm{Nd}$ & 17 & 34 \\
\hline & & & $\mathrm{Kh}$ & 9 & 18 \\
\hline & Extended PSC1 & 1000 & $\mathrm{Cc}$ & 8 & 16 \\
\hline
\end{tabular}

الجدول (6.1): مقارنة الخوارزميات المختلفة للتدرج المترافق 


\begin{tabular}{|c|c|c|c|c|c|}
\hline & & & $\begin{array}{l}\mathrm{Nd} \\
\mathrm{Kh}\end{array}$ & $\begin{array}{c}68 \\
8\end{array}$ & $\begin{array}{c}1945 \\
16\end{array}$ \\
\hline \multirow{6}{*}{6} & \multirow{3}{*}{ ENGVAL1 (CUTE) } & \multirow{3}{*}{100} & $\mathrm{Cc}$ & 26 & 48 \\
\hline & & & $\mathrm{Nd}$ & 38 & 71 \\
\hline & & & $\mathrm{Kh}$ & 28 & 51 \\
\hline & \multirow{3}{*}{ cENGVAL1 (CUTE) } & \multirow{3}{*}{1000} & $\mathrm{Cc}$ & 93 & 2429 \\
\hline & & & $\mathrm{Nd}$ & 108 & 2206 \\
\hline & & & $\mathrm{Kh}$ & 95 & 2205 \\
\hline \multirow{6}{*}{7} & \multirow{3}{*}{ BDQRTIC (CUTE) } & \multirow{3}{*}{100} & $\mathrm{Cc}$ & 472 & 10904 \\
\hline & & & $\mathrm{Nd}$ & 2002 & 2032 \\
\hline & & & $\mathrm{Kh}$ & 340 & 6313 \\
\hline & \multirow{3}{*}{ BDQRTIC (CUTE) } & \multirow{3}{*}{1000} & $\mathrm{Cc}$ & 2002 & 95072 \\
\hline & & & $\mathrm{Nd}$ & 2002 & 2026 \\
\hline & & & $\mathrm{Kh}$ & 2002 & 85662 \\
\hline \multirow{6}{*}{8} & \multirow{3}{*}{$\begin{array}{c}\text { Extended DENSCHNA } \\
\text { (CUTE) }\end{array}$} & \multirow{3}{*}{100} & $\mathrm{Cc}$ & 11 & 20 \\
\hline & & & $\mathrm{Nd}$ & 25 & 38 \\
\hline & & & $\mathrm{Kh}$ & 10 & 18 \\
\hline & \multirow{3}{*}{$\begin{array}{c}\text { Extended DENSCHNA } \\
\text { (CUTE) }\end{array}$} & \multirow{3}{*}{1000} & $\mathrm{Cc}$ & 22 & 35 \\
\hline & & & $\mathrm{Nd}$ & 24 & 38 \\
\hline & & & $\mathrm{Kh}$ & 10 & 19 \\
\hline \multirow{6}{*}{9} & \multirow{3}{*}{$\begin{array}{c}\text { Extended DENSCHNC } \\
\text { (CUTE) }\end{array}$} & \multirow{3}{*}{100} & $\mathrm{Cc}$ & 14 & 26 \\
\hline & & & $\mathrm{Nd}$ & 125 & 163 \\
\hline & & & $\mathrm{Kh}$ & 14 & 26 \\
\hline & \multirow{3}{*}{$\begin{array}{c}\text { Extended DENSCHNC } \\
\text { (CUTE) }\end{array}$} & \multirow{3}{*}{1000} & $\mathrm{Cc}$ & 14 & 27 \\
\hline & & & $\mathrm{Nd}$ & 133 & 171 \\
\hline & & & $\mathrm{Kh}$ & 13 & 24 \\
\hline \multirow{6}{*}{10} & & & $\mathrm{Cc}$ & 23 & 45 \\
\hline & Generalized & 100 & $\mathrm{Nd}$ & 37 & 63 \\
\hline & & & $\mathrm{Kh}$ & 25 & 43 \\
\hline & & & $\mathrm{Cc}$ & 29 & 169 \\
\hline & Generalized & 1000 & $\mathrm{Nd}$ & 52 & 463 \\
\hline & & & $\mathrm{Kh}$ & 30 & 113 \\
\hline & & & $\mathrm{Cc}$ & 11 & 22 \\
\hline & Extended Tridiagonal 1 & 100 & $\mathrm{Nd}$ & 2002 & 2102 \\
\hline 11 & & & $\mathrm{Kh}$ & 12 & 24 \\
\hline 11 & & & $\mathrm{Cc}$ & 15 & 28 \\
\hline & Extended Tridiagonal 1 & 1000 & $\mathrm{Nd}$ & 2002 & 2015 \\
\hline & & & $\mathrm{Kh}$ & 12 & 24 \\
\hline & & & $\mathrm{Cc}$ & 10 & 12 \\
\hline & Extended Himmelblau & 100 & $\mathrm{Nd}$ & 10 & 12 \\
\hline 12 & & & $\mathrm{Kh}$ & 10 & 12 \\
\hline 12 & & & $\mathrm{Cc}$ & 10 & 12 \\
\hline & Extended Himmelblau & 1000 & $\mathrm{Nd}$ & 10 & 12 \\
\hline & & & $\mathrm{Kh}$ & 10 & 12 \\
\hline & & & $\mathrm{Cc}$ & 23 & 47 \\
\hline & FLETCHCR (CUTE) & 100 & $\mathrm{Nd}$ & 39 & 79 \\
\hline & & & $\mathrm{Kh}$ & 24 & 48 \\
\hline 13 & & & $\mathrm{Cc}$ & 30 & 60 \\
\hline & FLETCHCR (CUTE) & 1000 & $\mathrm{Nd}$ & 33 & 63 \\
\hline & & & $\mathrm{Kh}$ & 31 & 56 \\
\hline 14 & Extended Block- & 100 & $\mathrm{Cc}$ & 14 & 24 \\
\hline & Diagonal BD2 & & $\mathrm{Nd}$ & 123 & 157 \\
\hline
\end{tabular}




\begin{tabular}{|c|c|c|c|c|c|}
\hline & & & $\mathrm{Kh}$ & 15 & 25 \\
\hline & $\begin{array}{l}\text { Extended Block- } \\
\text { Diagonal BD2 }\end{array}$ & 1000 & $\begin{array}{l}\mathrm{Cc} \\
\mathrm{Nd} \\
\mathrm{Kh}\end{array}$ & $\begin{array}{c}13 \\
131 \\
15\end{array}$ & $\begin{array}{c}24 \\
167 \\
27\end{array}$ \\
\hline \multirow{2}{*}{15} & $\begin{array}{c}\text { Extended } \\
\text { DENSCHNF(CUTE) }\end{array}$ & 100 & $\begin{array}{l}\mathrm{Cc} \\
\mathrm{Nd} \\
\mathrm{Kh}\end{array}$ & $\begin{array}{l}21 \\
20 \\
23\end{array}$ & $\begin{array}{l}37 \\
34 \\
37\end{array}$ \\
\hline & $\begin{array}{c}\text { Extended } \\
\text { DENSCHNF(CUTE) }\end{array}$ & 1000 & $\begin{array}{l}\mathrm{Cc} \\
\mathrm{Nd} \\
\mathrm{Kh}\end{array}$ & $\begin{array}{l}24 \\
22 \\
23\end{array}$ & $\begin{array}{l}44 \\
39 \\
38\end{array}$ \\
\hline
\end{tabular}
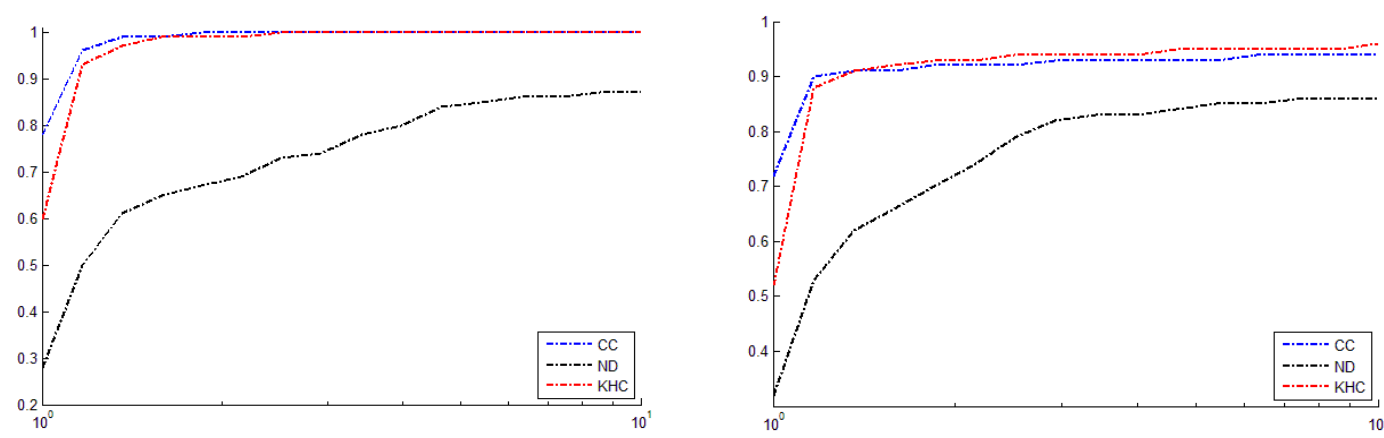

شكل (1) مقارنة بين الخوارزميات في عدد التكرارات

شكل (2) مقارنة بين الخوارزميات في عدد مرات حساب الدالة

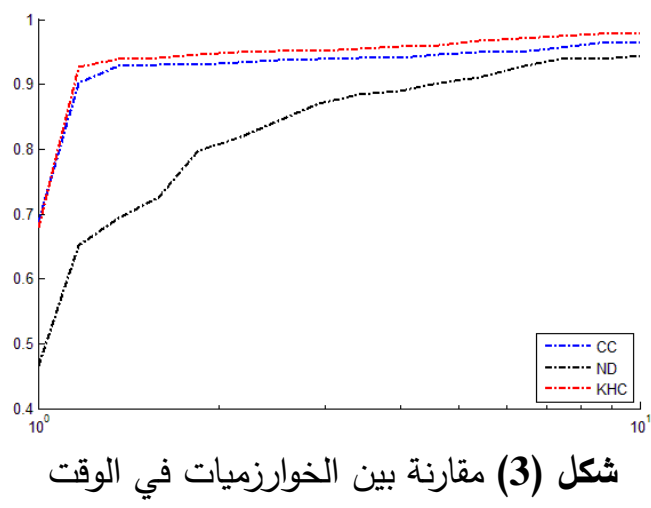


[1] Andrei, N., (2008), An Unconstrained Optimization Test Function collection, Advance Model. Optimization, Vol.(10), pp.147-161.

[2] Dai, Y. H. and Liao, L. Z., (2001), "New Conjugacy Conditions and Related Nonlinear Conjugate Gradient Methods". Applied Mathematics and Optimization, Springer-Verlag, New York, USA, 43, PP.87-101.

[3] Dolan.E. D and Mor'e.J. J, (2002), "Benchmarking optimization software with performance profiles", Math. Programming, 91, pp.201-213.

[4] Fletcher, R., (1987), Practical Methods of Optimization. Unconstrained Optimization, A Wiley-Interscience Publication, John Wiley \& Sons, Inc., New York, USA.

[5] Zoutendijk, G. (1970) "Nonlinear programming, computational methods" in Integer and Nonlinear Programming, J. Abadie (Ed.), North-Holland: Amsterdam, pp.37-86.

[6] Abbo, Khalil K, and Hisham M Khudhur. 2015a. "New A Hybrid Conjugate Gradient Fletcher-Reeves and Polak-Ribiere Algorithm for Unconstrained Optimization.” Tikrit Journal of Pure Science 21 (1).29-124.

[7] Abbo, Khalil K, and Hisham M Khudhur. 2015b. "New A Hybrid HestenesStiefel and Dai-Yuan Conjugate Gradient Algorithms for Unconstrained Optimization.” Tikrit Journal of Pure Science 21 (1).23-118.

[8] Abbo, Khalil K, Yoksal A Laylani, and Hisham M Khudhur. 2016. "Proposed New Scaled Conjugate Gradient Algorithm for Unconstrained Optimization" 5 (7).269-276.

[9] Abbo, Khalil K, Yoksal A Laylani, and Hisham M Khudhur. 2017. "A New Spectral Conjugate Gradient Algorithm Forunconstrained Optimization.”1-9.

www.tjprc.org .

[10] Hisham M. Khudhur. 2015. "Numerical and Analytical Study of Some Descent Algorithms to Solve Unconstrained Optimization Problems." University of Mosul College Computer Sciences and Mathematics Department of Mathematics Iraq. University of Mosul,Master Thesis.

[11] Jabbar, Hawraz N, Khalil K Abbo, and Hisham M Khudhur. 2018. "Four--Term Conjugate Gradient (CG) Method Based on Pure Conjugacy Condition for Unconstrained Optimization." Kirkuk University Journal for Scientific Studies 13 (2).13-101.

[12] Laylani, Yoksal A., Khalil K. Abbo, and Hisham M. Khudhur. 2018. "Training Feed Forward Neural Network with Modified Fletcher-Reeves Method." Journal Of Multidisciplinary Modeling And Optimization 1 (1).14-22. .http://dergipark.gov.tr/jmmo/issue/38716/392124\#article_cite

[13] Nocedal, J. and Wright, S. J., (2006), Numerical Optimization Springer Series in Operation Research, 2nd edition, Springer-Verlag, New York. 
[14] Sun, W. and Yuan, Y., (2006), Optimization Theory and Methods, Nonlinear Programming, Springer Science, Business Media, LIC., New York.

[15] Hestenes, M. R. and Stiefel, E., (1952), Methods of Conjugate Gradients for Solving Linear Systems, Journal of Research of the National Bureau of Standards, Vol. (5), No.(49), pp.409-436.

[16] Pedregal, P., (2004), Introducation to Optimization, Springer-Verlag, Inc., New York, USA.

[17] Powell, M. J. D., (1977), Restart Procedure for Conjugate Gradient Method, Mathematical Programming.12-241-254.

[18] Chong, E. K. P. and Zak, S. H., (2001), An Introduction to Optimization, Jone Wiley \& Sons, Inc., Canada.

[19] Dai, Y. H. and Yuan, Y., (1999), A Nonlinear Conjugate Gradient Method with a Strong Global Convergence Property, SIAM Journal on Optimization, 10 (1). $177-182$.

[20] Fletcher, R. and Reeves, C. M., (1964), "Function Minimization by Conjugate Gradients". Computer Journal.7,142-154. 